QUARTERLY OF APPLIED MATHEMATICS

VOLUME LXVII, NUMBER 4

DECEMBER 2009, PAGES 735-743

S 0033-569X(09)01161-8

Article electronically published on May 14, 2009

\title{
ENERGY BALANCE FOR VISCOELASTIC BODIES IN FRICTIONLESS CONTACT
}

\author{
BY \\ DAVID E. STEWART \\ Department of Mathematics, University of Iowa, Iowa City, Iowa 52242
}

\begin{abstract}
In this paper it is shown that the change in the energy for a linearly viscoelastic body (with Kelvin-Voigt type viscosity) in frictionless contact with a rigid obstacle can be accounted for by viscous losses and the work done by external forces. Thus there is no change in the energy due to impacts, unlike the case of rigid-body dynamics. The result can be extended to a wide class of dynamic viscoelastic boundary thin obstacle problems of similar type.
\end{abstract}

1. Introduction. The impact of an elastic or viscoelastic body against a rigid obstacle using the Signorini contact conditions has been the subject of many papers (see, for example, 3, 5, 17, 8, 9, 12, 13, 14, 15, 16, 17, 18, 19, 20, 21, 22, 24, 23). While most papers focus on showing existence of solutions, or numerical approximation of solutions to these problems, relatively little has been published regarding conservation of energy, or rigorously accounting for changes in the energy [18, 19, 21, 23. In particular, whether in general the contact forces for contact with a rigid obstacle do non-zero work on an elastic or visco-elastic body has been an open question. All the results related to conservation of energy or accounting for changes in energy so far have either made special assumptions about the governing equations or the geometry or both. The paper [18, which is perhaps the most sophisticated paper dealing with energy conservation or balance for impact-type problems, applies to the wave equation in a half-space $\mathbb{R}_{+} \times \mathbb{R}^{n-1}$. On the other hand, 21 is for a one-dimensional wave equation, but requires a convex obstacle, and also $\partial u / \partial t\left(x, t^{+}\right)=-\partial u / \partial t\left(x, t^{-}\right)$whenever $u(x, t)$ meets the obstacle. This corresponds to requiring a coefficient of restitution of one in a rigid-body setting. The paper [19] obtains an energy balance for a one-dimensional visco-elastic rod with impact on the boundary. Finally, 23 is able to obtain conservation of energy for elastic bodies in a modified impact law that artificially reduces the problem to make the contact forces finite-dimensional functions of time.

Received July 25, 2008.

2000 Mathematics Subject Classification. Primary 74M20; Secondary 35L85, 49J40.

Key words and phrases. Impact, viscoelasticity, energy balance, dynamic variational inequalities.

This work was supported in part by the NSF under grant DMS-0139709.

E-mail address: dstewart@math.uiowa.edu

(C)2009 Brown University 
This paper shows that the contact forces for a visco-elastic body in contact with a rigid obstacle do zero net work, and that the energy of the body can be accounted for solely by means of the external forces and viscous damping.

Obtaining energy balances is a difficult task. One of the difficulties is that while penalty methods can be developed so that energy is conserved in the penalty approximation, all the methods used to obtain a solution of the limiting problem (with Signorini contact conditions) must rely on weak convergence in the appropriate Sobolev spaces [7, 8, 9, 12, 13, 15, 16. The energy, being a convex function of the deformation and velocity fields, is at best a weakly lower semicontinuous function. The limiting solution cannot then be guaranteed to conserve energy or to satisfy an appropriate energy balance in the viscoelastic case.

This might be regarded as a mathematical curiosity, except that it has a clear physical interpretation: where there is weak but not strong convergence, more and more of the energy could be pushed into higher and higher frequency modes. Thus penalty approximations with very large penalty parameters (as might be suitable for describing contact at an atomic level) may result in significant amounts of energy being pushed into extremely high-frequency vibrations, which is indistinguishable from heat. That is, impacts could directly turn gross kinetic energy into heat. Any finite-dimensional simulation, using finite element methods for example, might keep this energy in elastic vibrations, albeit at frequencies well above those where the finite-dimensional approximations are valid.

Thus it is highly desirable to show that energy is conserved, or that changes in energy can be accounted for, without resorting to "energy losses due to impact". Since standard approaches rely on weak convergence in Sobolev spaces, some new tools are needed.

In a remarkable paper, Petrov and Schatzman [19] introduce some new tools. They consider the impact of a viscoelastic rod against a rigid obstacle at one end, and obtain an energy balance. The tools used by Petrov and Schatzman are convolution complementarity problems (see next paragraph); unfortunately this approach does not generalize to more than one dimension. However, it does give some insight into the mathematics of impact problems. One thing that should be noted from [19] is that incorporating viscosity makes the deformation field more regular but the contact forces less regular.

Results on conservation of energy or energy balances have been established via convolution complementarity problems $[19,23$. These are complementarity problems of the form: Given $k: \mathbb{R}_{+} \rightarrow \mathbb{R}^{n \times n}$ and $q: \mathbb{R}_{+} \rightarrow \mathbb{R}^{n}$, find $z: \mathbb{R}_{+} \rightarrow \mathbb{R}^{n}$ satisfying

$$
0 \leq z(t) \quad \perp \quad(k * z)(t)+q(t) \geq 0 \quad \text { for all } t \geq 0,
$$

where " $a \geq 0$ " for a vector $a \in \mathbb{R}^{n}$ means that " $a_{i} \geq 0$ for $i=1,2, \ldots, n$ ", $k * z$ is the convolution

$$
(k * z)(t)=\int_{0}^{t} k(s) z(t-s) d s,
$$

and " $a \perp b$ " means that $a^{T} b=0$. In the context of mechanical impact problems, $z$ typically represents the normal contact force, while $k * z+q$ represents the gap between the body (or bodies) and the obstacle. If we set $w=k * z+q$, then provided $w$ is sufficiently regular, not only is $z(t)^{T} w(t)=0$ for all $t$, but $z(t)^{T} d w / d t(t)=0$ for (almost) all $t$ as 
well [23, 24]. From this property, energy balances can be proved 23, 24, including ones for the viscoelastic rod problem of Petrov and Schatzman.

However, in dealing with most boundary thin obstacle problems, the normal contact force is removed from the problem by formulating it as a variational inequality [7, 8, 9 , 12, 13, 15, 16] rather than as a complementarity problem as is done in [1, 2]. Variational inequalities [4, 11] have the form: Given $F: X \rightarrow X^{*}$ and a closed convex set $K \subseteq X$, find $u \in X$ such that

$$
\langle\widetilde{u}-u, F(u)\rangle \geq 0 \quad \text { for all } \widetilde{u} \in K
$$

If $K$ is also a cone (that is, $x \in K$ implies $\alpha x \in K$ for any $\alpha \geq 0$ ), then (1.1) is equivalent to a generalized complementarity problem: Find $u$ such that

$$
K \ni u \quad \perp \quad F(u) \in K^{*},
$$

where $K^{*}=\{z \mid\langle z, w\rangle \geq 0$ for all $w \in K\}$ is the dual cone to $K$, and " $a \perp b$ " means that $\langle a, b\rangle=0$.

Complementarity formulations must explicitly invoke the normal contact force, and the difficulty with these formulations for impact problems is in obtaining suitable bounds for these contact forces. This is dealt with successfully in [1, 2, by restricting attention to problems in which the cone $K$ is strongly pointed in the relevant Sobolev space. A cone $K$ in a Banach space $X$ is strongly pointed if there is $\delta>0$ and $\xi \in X^{*}$ where $\langle\xi, x\rangle \geq \delta\|x\|$ for all $x \in K$, where $\langle\cdot, \cdot\rangle$ is the duality pairing between $X$ and its dual space $X^{*}$. The cone $K$ being strongly pointed is equivalent to $K^{*}$ being solid in $X^{*}$; that is, $K^{*}$ has nonempty interior. This property fails for elasticity problems in more than one dimension. Since bounds on the contact forces are not available, a common approach to treating impact problems is to use variational inequalities that do not need to explicitly refer to the normal contact forces at all $[3,5,7,8,9,12,13,14,15,16,17,18,19,20,21,22,24,23]$.

Energy conservation results have been obtained using complementarity formulations [19, 23]. These results have been obtained by developing lemmas on "differentiating complementarity problems": showing that if $K \ni a(t) \perp b(t) \in K^{*}$ for sufficiently regular $a$ and $b$, then $a^{\prime}(t) \perp b(t)$ for almost all $t$.

The approach taken in this paper is to start with the variational inequality formulation, and from that obtain a result on "differentiating variational inequalities" analogous to the results in [24]. From that, regularity results that have already been established for viscoelastic bodies in impact, and some technical considerations, we are able to conclude with an energy balance for viscoelastic bodies.

2. Differentiating variational inequalities. The main result for this section is:

Lemma 2.1. Let $K$ be a closed, convex set in a separable reflexive Banach space $X$, $f:[0, T] \rightarrow X^{*}, f \in L^{p}\left(0, T ; X^{*}\right)$, and $u:[0, T] \rightarrow X$ be a solution to the variational inequality: $u(t) \in K$ for all $t$, and

$$
\int_{0}^{T}\langle\widetilde{u}(t)-u(t), f(t)\rangle d t \geq 0 \quad \text { for all continuous } \widetilde{u}:[0, T] \rightarrow K .
$$

Provided the distributional derivative $u^{\prime} \in L^{q}(0, T ; X)(1 / p+1 / q=1)$, then $\left\langle u^{\prime}(t), f(t)\right\rangle$ $=0$ for almost all $t \in[0, T]$. 
Proof. First we note that since the space of continuous functions $[0, T] \rightarrow X$ is dense in the space $L^{\infty}(0, T ; X)$, (2.1) holds for all $\widetilde{u} \in L^{\infty}(0, T ; X)$. For any measurable set $E$ and $w \in K$ we can let $\widetilde{u}(t)=u(t)$ for $t \notin E$ and $\widetilde{u}(t)=w$ for $t \in E$; we have

$$
\int_{E}\langle w-u(t), f(t)\rangle d t \geq 0 .
$$

Thus there is a Lebesgue null set $N_{w}$ such that $\langle w-u(t), f(t)\rangle \geq 0$ for all $t \notin N_{w}$. As $X$ is reflexive, there is a countable dense subset $\left\{w_{i}\right\}_{i=1}^{\infty}$; setting $N=\bigcup_{i=1}^{\infty} N_{w_{i}}$, which is also a Lebesgue null set, we see that

$$
\langle w-u(t), f(t)\rangle \geq 0 \quad \text { for all } w \in K \text { and } t \notin N .
$$

On the other hand, by standard arguments, since $v:=u^{\prime} \in L^{q}(0, T ; X), u$ must be absolutely continuous, and as $X$ has the Radon-Nikodym property [10, Thm. III.3.1], $u(t)=u(s)+\int_{s}^{t} v(\tau) d \tau$ for all $t>s$ [10, Thm. IV.3.2]. Therefore, the ordinary derivative $\lim _{h \rightarrow 0}(u(t+h)-u(t)) / h$ exists and is equal to $v(t)$ for almost all $t$ [10, Thm. II.2.9]. Let $F$ be the set of $t \in[0, T]$ where either $\lim _{h \rightarrow 0}(u(t+h)-u(t)) / h$ fails to exist, or exists but is different from $v(t)$. This is also a null set.

Then on the set $[0, T] \backslash(N \cup F)$ we have

$$
\left\langle f(t), u^{\prime}(t)\right\rangle=\lim _{h \rightarrow 0}\left\langle f(t), \frac{u(t+h)-u(t)}{h}\right\rangle .
$$

But,

$$
\begin{aligned}
& \left\langle f(t), \frac{u(t+h)-u(t)}{h}\right\rangle \geq 0 \quad \text { for } h>0, \text { and } \\
& \left\langle f(t), \frac{u(t+h)-u(t)}{h}\right\rangle \leq 0 \quad \text { for } h<0 .
\end{aligned}
$$

Thus the limit must be zero on $[0, T] \backslash(N \cup F)$. Noting that $t \mapsto\left\langle f(t), u^{\prime}(t)\right\rangle$ is in $L^{1}(0, T)$ and that $\left\langle f(t), u^{\prime}(t)\right\rangle=0$ for almost all $t$, we see that $\left\langle f, u^{\prime}\right\rangle=0$ in $L^{1}(0, T)$ and in the sense of measures.

This result is not particularly sharp: any $X$ with separable dual and that has the Radon-Nikodym property would suffice. Analogous results for dynamic complementarity problems are shown in 24].

There is a result for two derivatives.

Lemma 2.2. Let $K$ be a closed, convex set in a reflexive Banach space $X, f:[0, T] \rightarrow X^{*}$, $f \in W^{1, p}\left(0, T ; X^{*}\right)$, and let $u:[0, T] \rightarrow X$ be a solution to the variational inequality: $u(t) \in K$ for all $t$, and

$$
\langle\widetilde{u}-u(t), f(t)\rangle \geq 0 \quad \text { for all } \widetilde{u} \in K .
$$

Then provided $u \in W^{1, q}(0, T ; X)(1 / p+1 / q=1),\left\langle u^{\prime}(t), f^{\prime}(t)\right\rangle \leq 0$ for almost all $t \in[0, T]$.

Proof. As in Lemma 2.1. both $u^{\prime}(t)=\lim _{h \rightarrow 0}(u(t+h)-u(t)) / h$ and $f^{\prime}(t)=$ $\lim _{h \rightarrow 0}(f(t+h)-f(t)) / h$ exist for almost all $t$. For any $t$ where both are true, for 
$h>0$, from the definition of a solution of the VI,

$$
\begin{aligned}
& 0 \geq\left\langle f(t+h), \frac{u(t+h)-u(t)}{h}\right\rangle, \\
& 0 \leq\left\langle f(t), \frac{u(t+h)-u(t)}{h}\right\rangle .
\end{aligned}
$$

Subtracting, we get

$$
0 \geq\left\langle\frac{f(t+h)-f(t)}{h}, \frac{u(t+h)-u(t)}{h}\right\rangle .
$$

Alternatively, if $h<0$, let $h^{\prime}=-h>0$ and $t^{\prime}=t+h$. Then applying the previous argument gives

$$
0 \geq\left\langle\frac{f\left(t^{\prime}+h^{\prime}\right)-f\left(t^{\prime}\right)}{h^{\prime}}, \frac{u\left(t^{\prime}+h^{\prime}\right)-u\left(t^{\prime}\right)}{h^{\prime}}\right\rangle
$$

That is,

$$
\begin{aligned}
0 & \geq\left\langle\frac{f(t)-f(t+h)}{-h}, \frac{u(t)-u(t+h)}{-h}\right\rangle \\
& =\left\langle\frac{f(t+h)-f(t)}{h}, \frac{u(t+h)-u(t)}{h}\right\rangle
\end{aligned}
$$

In either case, taking $h \rightarrow 0$ for any $t$ where both derivatives exist gives

$$
0 \geq\left\langle f^{\prime}(t), u^{\prime}(t)\right\rangle,
$$

as desired.

3. An energy balance for viscoelastic bodies in impact. In Kuttler and Shillor [16, the problem of contact of a viscoelastic body with a rigid obstacle with a bounded (undeformed) domain $\Omega \subset \mathbb{R}^{n}$ is considered, with the boundary $\partial \Omega$ divided into three regions: $\Gamma_{1}$ on which $\mathbf{u}(t, \mathbf{x})=\mathbf{g}(\mathbf{x})$ is given (fixed boundary), $\Gamma_{2}$ on which $\sigma(t, \mathbf{x}) \mathbf{n}(\mathbf{x})=$ $\mathbf{h}(t, \mathbf{x})$ is given (given traction forces), and $\Gamma_{3}$ on which contact can occur, where

$$
\begin{aligned}
\rho \frac{\partial^{2} \mathbf{u}}{\partial t^{2}} & =\operatorname{div} \sigma(t, \mathbf{x})+\mathbf{f}(t, \mathbf{x}) \quad \text { in } \Omega \\
\sigma(t, \mathbf{x}) & =A \varepsilon(\mathbf{u})+B \varepsilon(\dot{\mathbf{u}}) \quad \text { is the stress tensor, } \\
\varepsilon(t, \mathbf{x}) & =\frac{1}{2}\left(\nabla \mathbf{u}(t, \mathbf{x})+\nabla \mathbf{u}(t, \mathbf{x})^{T}\right) \quad \text { is the strain tensor, } \\
\sigma(t, \mathbf{x}) \mathbf{n}(\mathbf{x}) & =N(t, \mathbf{x}) \mathbf{n}(\mathbf{x}) \quad \text { on } \Gamma_{3}, \\
0 \leq \mathbf{n}(\mathbf{x}) \cdot \mathbf{u}(t, \mathbf{x})-\gamma(\mathbf{x}) & \perp N(t, \mathbf{x}) \geq 0 \quad \text { on } \Gamma_{3} .
\end{aligned}
$$

Assuming that $\rho$ is constant, we can scale time so that $\rho \equiv 1$. Note that unlike the rigidbody case there is no coefficient of restitution. For now we will assume that $\mathbf{g}(\mathbf{x})=0$ on $\Gamma_{1}$ for simplicity of the development. We introduce the following function spaces:

$$
\begin{aligned}
V & =\left\{\mathbf{w} \in H^{1}(\Omega)^{d}|\mathbf{w}| \Gamma_{1}=\mathbf{0}\right\} \\
H & =\mathbf{w} \in L^{2}(\Omega)^{d} .
\end{aligned}
$$


We denote the dual of a Banach space $X$ as $X^{\prime}$. We consider the spaces as forming a Gelfand triple $V \subset H=H^{\prime} \subset V^{\prime}$ with compact inclusions. Kuttler and Shillor transform this into a variational inequality: Let

$\mathcal{K}=\left\{\mathbf{w} \in L^{2}\left(0, T ; H^{1}(\Omega)\right)|\mathbf{w}| \Gamma_{1}=\mathbf{0}, \mathbf{n}(\mathbf{x}) \cdot \mathbf{w}(t, \mathbf{x})-\gamma(\mathbf{x}) \geq 0\right.$ for all $\left.\mathbf{x} \in \Gamma_{3}, t>0\right\}$.

The operators $L$ and $M$ are given by $L \mathbf{w}=-\operatorname{div}(A \varepsilon(\mathbf{w}))$ and $M \mathbf{w}=-\operatorname{div}(B \varepsilon(\mathbf{w}))$. Note that $A$ and $B$ map symmetric $d \times d$ matrices into symmetric $d \times d$ matrices, and for any $d \times d$ symmetric matrix $\Xi,\langle\Xi, A \Xi\rangle:=\sum_{i, j} \Xi_{i j}(A \Xi)_{i j} \geq \eta_{A}\langle\Xi, \Xi\rangle$ with $\eta_{A}>0$, with a corresponding inequality for $B$. Then $L$ and $M$ are elliptic partial differential operators. The dynamic contact problem can be re-written as a variational inequality where $\mathbf{u} \in \mathcal{K}$ and

$$
\int_{0}^{T}\left\langle\frac{d \mathbf{v}}{d t}+L \mathbf{u}+M \mathbf{v}-\mathbf{f}, \mathbf{u}-\mathbf{w}\right\rangle d t \geq 0 \text { for all } \mathbf{w} \in \mathcal{K}
$$

with $\mathbf{v}=d \mathbf{u} / d t$. Kuttler and Shillor apply integration by parts to $\int_{0}^{T}\langle d \mathbf{v} / d t, \mathbf{u}-\mathbf{w}\rangle d t$ to give

$$
\langle\mathbf{v}(T), \mathbf{u}(T)-\mathbf{w}(T)\rangle-\langle\mathbf{v}(0), \mathbf{u}(0)-\mathbf{w}(0)\rangle-\int_{0}^{T}\left\langle\mathbf{v}, \mathbf{v}-\mathbf{w}^{\prime}\right\rangle d t .
$$

Kuttler and Shillor then use penalty approximations (with penalty parameter $\epsilon \downarrow 0$ ) to obtain a sequence of approximate solutions $\mathbf{u}_{\epsilon}, \mathbf{v}_{\epsilon}=d \mathbf{u}_{\epsilon} / d t$ with the following properties:

$$
\begin{aligned}
& \mathbf{u}_{\epsilon} \text { uniformly bounded in } L^{\infty}(0, T ; V), \\
& \mathbf{v}_{\epsilon} \text { uniformly bounded in } L^{\infty}(0, T ; H) \cap L^{2}(0, T ; V), \\
& \mathbf{v}_{\epsilon}^{\prime} \text { uniformly bounded in } L^{2}\left(0, T ; V^{\prime}\right) .
\end{aligned}
$$

The uniform bounds on $\mathbf{v}_{\epsilon}$ mean that the $\mathbf{v}_{\epsilon}$ lie in a compact subset of $L^{2}\left(0, T ;[H, V]_{\theta}\right)$ for any $0<\theta<1$, where $[H, V]_{\theta}$ is the interpolation space of $H$ and $V$ [6, 25]. This means that $\int_{0}^{T}\left\langle\mathbf{v}_{\epsilon}, \mathbf{v}_{\epsilon}\right\rangle d t \rightarrow \int_{0}^{T}\langle\mathbf{v}, \mathbf{v}\rangle d t$ in a suitable subsequence.

A technical difficulty is to show that $t \mapsto \frac{1}{2}\langle\mathbf{v}(t), \mathbf{v}(t)\rangle$ is absolutely continuous, and that $(d / d t) \frac{1}{2}\langle\mathbf{v}(t), \mathbf{v}(t)\rangle=\left\langle\mathbf{v}(t), \mathbf{v}^{\prime}(t)\right\rangle$ for almost all $t$. If $\mathbf{v}$ is absolutely continuous, this is standard. However, we will show this below without showing that $\mathbf{v}$ is absolutely continuous $[0, T] \rightarrow H$. Let $\left\{\phi_{i}\right\}_{i=1}^{\infty}$ be an $H$-orthonormal basis of eigenfunctions of $I+L$, and write $\mathbf{v}(t)=\sum_{i} v_{i}(t) \phi_{i}$. Since $\mathbf{v}^{\prime} \in L^{2}\left(0, T ; V^{\prime}\right)$, this means that each $v_{i}^{\prime}$ is in $L^{2}(0, T)$ and $v_{i}$ is absolutely continuous. Then the ordinary derivative $v_{i}^{\prime}$ exists almost everywhere. Thus $v_{i} v_{i}^{\prime}$ is integrable on $[0, T]$, and we can apply the standard rules to show that $v_{i}^{2}$ is absolutely continuous and $\left(\frac{1}{2} v_{i}^{2}\right)^{\prime}=v_{i} v_{i}^{\prime}$ almost everywhere. We can choose an equivalent norm for $V:\|\mathbf{v}\|_{V}^{2}=\sum_{i} \lambda_{i} v_{i}^{2}$, where $(I+L) \phi_{i}=\lambda_{i} \phi_{i}$ for all $i$. Then we have a corresponding equivalent norm for $V^{\prime}:\|\mathbf{w}\|_{V^{\prime}}^{2}=\sum_{i} \lambda_{i}^{-1} w_{i}^{2}$. We show that $\sum_{i}\left(\frac{1}{2} v_{i}^{2}\right)^{\prime}$ is integrable as follows: $\left|v_{i}\right|\left|v_{i}^{\prime}\right| \leq \frac{1}{2}\left(\lambda_{i} v_{i}^{2}+\lambda_{i}^{-1}\left(v_{i}^{\prime}\right)^{2}\right)$; summing over $i$ gives $\sum_{i}\left|v_{i}(t) v_{i}^{\prime}(t)\right| \leq$ $\frac{1}{2}\left(\|\mathbf{v}(t)\|_{V}^{2}+\left\|\mathbf{v}^{\prime}(t)\right\|_{V^{\prime}}^{2}\right)$, which is integrable. So $\sum_{i=1}^{N} v_{i}(t) v_{i}^{\prime}(t)$ is integrable, uniformly in $N$. Now for any $0 \leq t \leq T, \int_{0}^{t} \sum_{i=1}^{N} v_{i}(s) v_{i}^{\prime}(s) d s=\frac{1}{2} \sum_{i=1}^{N} v_{i}(t)^{2}-\frac{1}{2} \sum_{i=1}^{N} v_{i}(0)^{2}$. Using the dominated convergence theorem together with the bounds on $\|\mathbf{v}(t)\|_{H}$ and $\|\mathbf{v}(0)\|_{H}$ coming from $\mathbf{v}(0) \in V \subset H$ and $\mathbf{v} \in L^{\infty}(0, T ; H)$, we can take the limit for 
almost every $t$ to get

$$
\int_{0}^{t}\left\langle\mathbf{v}(s), \mathbf{v}^{\prime}(s)\right\rangle d s=\frac{1}{2}\langle\mathbf{v}(t), \mathbf{v}(t)\rangle-\frac{1}{2}\langle\mathbf{v}(0), \mathbf{v}(0)\rangle
$$

for almost all $t$. Since $\left\langle\mathbf{v}, \mathbf{v}^{\prime}\right\rangle \in L^{1}(0, T)$, we have $t \mapsto \frac{1}{2}\langle\mathbf{v}(t), \mathbf{v}(t)\rangle$ is an absolutely continuous function (possibly after redefinition on a null set), and $(d / d t) \frac{1}{2}\langle\mathbf{v}(t), \mathbf{v}(t)\rangle=$ $\left\langle\mathbf{v}(t), \mathbf{v}^{\prime}(t)\right\rangle$ for almost all $t$. Note that in the same way, it can be shown that $(d / d t) \frac{1}{2}\langle L \mathbf{u}(t), \mathbf{u}(t)\rangle=\langle L \mathbf{u}(t), \mathbf{v}(t)\rangle$ for almost all $t$.

Since $\mathbf{v} \in L^{2}(0, T ; V)$ and $\mathbf{v}^{\prime} \in L^{2}\left(0, T ; V^{\prime}\right)$, we can apply integration by parts to obtain

$$
\int_{a}^{b}\left\langle\frac{d \mathbf{v}}{d t}+L \mathbf{u}+M \mathbf{v}-\mathbf{f}, \mathbf{u}-\mathbf{w}\right\rangle d t \geq 0 \text { for all } \mathbf{w} \in \mathcal{K}
$$

for all $0 \leq a \leq b \leq T$. Applying the differentiation lemma above, Lemma 2.1, we obtain

$$
\left\langle\frac{d \mathbf{v}}{d t}+L \mathbf{u}+M \mathbf{v}-\mathbf{f}, \mathbf{v}\right\rangle=0 \quad \text { for almost all } t .
$$

Noting that $\langle d \mathbf{v} / d t, \mathbf{v}\rangle,\langle L \mathbf{u}, \mathbf{v}\rangle,\langle M \mathbf{v}, \mathbf{v}\rangle$, and $\langle\mathbf{f}, \mathbf{v}\rangle$ are all in $L^{1}(0, T)$, and applying integration by parts, we get

$$
\frac{d}{d t}\left(\frac{1}{2}\langle\mathbf{v}, \mathbf{v}\rangle+\frac{1}{2}\langle L \mathbf{u}, \mathbf{u}\rangle\right)=\langle\mathbf{f}, \mathbf{v}\rangle-\langle M \mathbf{v}, \mathbf{v}\rangle,
$$

where the function differentiated is absolutely continuous. We identify $\frac{1}{2}\langle\mathbf{v}, \mathbf{v}\rangle+\frac{1}{2}\langle L \mathbf{u}, \mathbf{u}\rangle$ as the total energy of the elastic body (kinetic plus elastic potential energy), $\langle\mathbf{f}, \mathbf{v}\rangle$ as the rate at which work is done by the external forces, and $\langle M \mathbf{v}, \mathbf{v}\rangle$ as the rate of energy loss due to viscosity. Thus we have an energy balance for linearly viscoelastic bodies in frictionless contact with a rigid obstacle.

4. Other examples. This approach can be applied to a wide range of boundary-thin impact problems with Kelvin-Voigt viscoelasticity. For example, consider the problem of a viscoelastic Euler-Bernoulli beam impacting at one end. Here we use subscripts to indicate partial derivatives:

$$
\begin{aligned}
\rho A u_{t t} & =-E I u_{x x x x}-E^{\prime} I u_{t x x x x}+f(t, x), \quad x \in(0, \ell), \quad t>0, \\
N(t) & =E I u_{x x x}(\ell, t)+E^{\prime} I u_{t x x x}(\ell, t), \\
0 & =E I u_{x x}(\ell, t)+E^{\prime} I u_{t x x}(\ell, t), \\
0 \leq N(t) & \perp u(\ell, t) \geq 0, \\
u(0, t) & =0, \quad u_{x}(0, t)=0 .
\end{aligned}
$$

Here $A$ is the cross-sectional area of the beam, $\rho$ is the mass density (mass per unit volume), $I$ is the second moment of area, $E$ is Young's modulus, $E^{\prime}$ is the corresponding quantity for viscosity, and $\ell$ is the length of the beam. In this case we set $H=L^{2}(0, \ell)$ and $V=H_{c f}^{2}(0, \ell)=\left\{u \in H^{2}(0, \ell) \mid u(0)=u^{\prime}(0)=0\right\}$ (the subscript " $c f$ " indicates "clamped at $x=0$ and free at $x=\ell ")$. The elasticity operator is $\mathcal{A}=E I\left(\partial^{4} / \partial x^{4}\right)$ and 
the viscosity operator is $\mathcal{B}=E^{\prime} I\left(\partial^{4} / \partial x^{4}\right)$, so that the equations become

$$
\rho A u_{t t}=-\mathcal{A} u-\mathcal{B} u_{t}+f(t, x) \quad \text { in }(0, \ell) .
$$

We assume $\rho$ and $A$ are time-independent, smooth, and bounded away from zero.

If we let $K=\left\{u \in H_{c f}^{2}(0, \ell) \mid u(\ell) \geq 0\right\}$, then the impact problem is equivalent to the variational inequality: Find $u:[0, T] \rightarrow K$ such that

$$
\int_{0}^{T}\left\langle\rho A u_{t t}+\mathcal{A} u+\mathcal{B} u_{t}-f(t), w(t)-u(t)\right\rangle_{V^{\prime} \times V} d t \geq 0
$$

for all continuous $w:[0, T] \rightarrow K$. We assume that $f \in L^{2}(0, T ; H)$. By standard methods, such as penalty methods, solutions can be shown to exist for this problem [17] with $u \in W^{1,2}(0, T ; V)$. We can show that $u_{t t} \in L^{2}\left(0, T ; V^{\prime}\right)$ as follows (using an argument in [16]): Take any $\phi \in C_{0}^{\infty}((0, T) \times \Omega)$ so that the support of $\phi$ is strictly contained within $\Omega$. Then since the contact force is only applied on the boundary,

$$
\begin{aligned}
\left|\int_{0}^{T} \int_{\Omega}\left[\rho A u_{t t}+\mathcal{A} u+\mathcal{B} u_{t}\right] \phi d V d t\right| & =\left|\int_{0}^{T} \int_{\Omega} f(t, x) \phi(t, x) d V(x) d t\right| \\
& \leq\|f\|_{L^{2}(0, T ; H)}\|\phi\|_{L^{2}(0, T ; H)} .
\end{aligned}
$$

Since $C_{0}^{\infty}((0, T) \times \Omega)$ is dense in $L^{2}((0, T) \times \Omega)=L^{2}(0, T ; H)$, this means that

$$
\left\|\rho A u_{t t}+\mathcal{A} u+\mathcal{B} u_{t}\right\|_{L^{2}(0, T ; H)} \leq\|f\|_{L^{2}(0, T ; H)} .
$$

Now $\mathcal{A} u \in L^{\infty}\left(0, T ; V^{\prime}\right)$ (since $\left.u \in L^{\infty}(0, T ; V)\right)$ and $\mathcal{B} u_{t} \in L^{2}\left(0, T ; V^{\prime}\right)$ (since $u_{t} \in$ $\left.L^{2}(0, T ; V)\right)$, so $\rho A u_{t t} \in L^{2}\left(0, T ; V^{\prime}\right)$. Since $\rho A$ is bounded away from zero and is smooth, $u_{t t} \in L^{2}\left(0, T ; V^{\prime}\right)$.

Thus we can apply Lemma 2.1 to obtain

$$
\left\langle\rho A u_{t t}+\mathcal{A} u+\mathcal{B} u_{t}-f(t), u_{t}\right\rangle_{V^{\prime} \times V}=0 \quad \text { almost everywhere. }
$$

Applying integration by parts (following the methods of the previous section) we find that for any $t$ at which the classical derivative $u_{t}$ is defined,

$$
\begin{aligned}
\frac{1}{2}\left\langle\rho A u_{t}(t), u_{t}(t)\right\rangle+\frac{1}{2}\langle\mathcal{A} u(t), u(t)\rangle \\
=\frac{1}{2}\left\langle\rho A u_{t}(0), u(0)\right\rangle+\frac{1}{2}\langle\mathcal{A} u(0), u(0)\rangle \\
\quad+\int_{0}^{t}\left\langle f(\tau), u_{t}(\tau)\right\rangle d \tau-\int_{0}^{t}\left\langle\mathcal{B} u_{t}(\tau), u_{t}(\tau)\right\rangle d \tau .
\end{aligned}
$$

That is, we have an energy balance. The technique, clearly, can be applied to a wide range of obstacle problems where the contact conditions are applied to a part of the boundary of the domain of the governing partial differential equation.

\section{REFERENCES}

[1] Jeongho Ahn and David E. Stewart. An Euler-Bernoulli beam with dynamic contact: Discretization, convergence and numerical results. SIAM J. Numer. Anal., 43(4):1455-1480, 2005. MR2182136 (2006e:35319)

[2] Jeongho Ahn and David E. Stewart. Existence of solutions for a class of impact problems without viscosity. SIAM J. Mathematical Analysis, 38(1):37-63, 2006. MR.2217307 (2007g:35158) 
[3] Kevin T. Andrews, M. Shillor, and S. Wright. On the dynamic vibrations of an elastic beam in frictional contact with a rigid obstacle. J. Elasticity, 42(1):1-30, 1996. MR1390198 (97h:73073)

[4] C. Baiocchi and A. Capelo. Variational and Quasivariational Inequalities: Applications to Free Boundary Problems. Wiley, Chichester, New York, 1984. MR745619 (86e:49018)

[5] A. Bamberger and M. Schatzman. New results on the vibrating string with a continuous obstacle. SIAM J. Math. Anal., 14(3):560-595, 1983. MR697529 (84g:35106)

[6] J. Bergh and J. Löfström. Interpolation Spaces, volume 223 of Grundlehren der mathmatischen Wissenschaften. Springer-Verlag, Berlin, Heidelberg, New York, 1976.

[7] Marius Cocu. Existence of solutions of Signorini problems with friction. Internat. J. Engrg. Sci., 22(5):567-575, 1984. MR750004 (86g:73050)

[8] Marius Cocu and Jean-Marc Ricaud. Existence results for a class of implicit evolution inequalities and applications to dynamic unilateral contact problems with friction. C. R. Acad. Sci. Paris, Sér. I, 329:839-844, 1999. MR 1724551(2000i:34122)

[9] Marius Cocu and Jean-Marc Ricaud. Analysis of a class of implicit evolution inequalities associated to viscoelastic dynamic contact problems with friction. Internat. J. Engrg. Sci., 38(14):1535-1552, 2000. MR1763039 (2001e:74066)

[10] J. Diestel and J. J. Uhl, Jr. Vector Measures, volume 15 of Mathematical Surveys. Amer. Math. Soc., Providence, RI, 1977. MR0453964 (56:12216)

[11] F. Facchinei and J.-S. Pang. Finite-Dimensional Variational Inequalities and Complementarity Problems I. Springer Series in Operations Research. Springer, New York, 2003.

[12] J. Jarušek and C. Eck. Dynamic contact problems with small Coulomb friction for viscoelastic bodies. Existence of solutions. Math. Models Methods Appl. Sci., 9(1):11-34, 1999. MR1671535 (2000b:74058)

[13] Jiří Jarušek and Christof Eck. Dynamic contact problems with friction in linear viscoelasticity. C. R. Acad. Sci. Paris Sér. I Math., 322(5):497-502, 1996. MR1381792 (97f:73044)

[14] N. Kikuchi and J. T. Oden. Contact problems in elasticity: a study of variational inequalities and finite element methods, volume 8 of SIAM Studies in Applied Mathematics. Society for Industrial and Applied Mathematics (SIAM), Philadelphia, PA, 1988. MR961258 (89j:73097)

[15] Jong Uhn Kim. A boundary thin obstacle problem for a wave equation. Commun. Partial Differential Equations, 14(8\&9):1011-1026, 1989. MR1017060(91a:35121)

[16] Kenneth Kuttler and Meir Shillor. Dynamic contact with Signorini's condition and slip rate dependent friction. Electron. J. Differential Equations, No. 83, 21 pp. (electronic), 2004. MR2075422 (2005d:74026)

[17] Kenneth L. Kuttler and Meir Shillor. Vibrations of a beam between two stops. Dyn. Contin. Discrete Impuls. Syst. Ser. B Appl. Algorithms, 8(1):93-110, 2001. MR1824287 (2003a:74023)

[18] G. Lebeau and M. Schatzman. A wave problem in a half-space with a unilateral constraint at the boundary. J. Differential Equations, 53:309-361, 1984. MR752204 (86b:35108)

[19] Adrien Petrov and Michelle Schatzman. Viscoélastodynamique monodimensionnelle avec conditions de Signorini. C. R. Math. Acad. Sci. Paris, 334(11):983-988, 2002. MR.1913722 (2003e:35214)

[20] E. J. Routh. A Treatise on the Dynamics of a System of Rigid Bodies. MacMillan, London, 1860.

[21] Michelle Schatzman. A hyperbolic problem of second order with unilateral constraints: the vibrating string with a concave obstacle. J. Math. Anal. Appl., 73(1):138-191, 1980. MR/560941(81d:35047)

[22] Michelle Schatzman and Michel Bercovier. Numerical approximation of a wave equation with unilateral constraints. Math. Comp., 53(187):55-79, 1989. MR969491 (89k:65112)

[23] David E. Stewart. Convolution complementarity problems with application to impact problems. IMA J. Applied Math., 71(1):92-119, 2006. MR2203045 (2007f:90117)

[24] David E. Stewart. Differentiating complementarity problems and fractional index convolution complementarity problems. Houston J. Mathematics, 33(1):301-322, 2006. MR2287857 (2008b:90128)

[25] Hans Triebel. Interpolation Theory, Function Spaces, Differential Operators. North-Holland, Amsterdam, New York, 1978. MR503903 (80i:46032b) 